\title{
Application of Scalable Visual Sensitivity Profile in Image and Video Coding
}

\author{
Qian Chen, Guangtao Zhai, Xiaokang Yang, and Wenjun Zhang \\ Institute of Image Communication and Information Processing \\ Shanghai Jiao Tong University, Shanghai, 200240, China \\ Email: \{qianchen, zhaiguangtao, xkyang, zhangwenjun\}@sjtu.edu.cn
}

\begin{abstract}
Computational visual attention models have facilitated various aspects of the evolution in visual communication systems. In this paper, we apply the proposed computational model for scalable visual sensitivity profile (SVSP) to image/video processing. SVSP generates a hierarchy of saliency maps that simulate the bottom-up and top-down attention of the human visual system (HVS). It can detect the most significant visual information in a picture and spatially prioritize the information to augment the visual quality, such as in noise-shaping and region-of-interest (ROI) coding of JPEG2000. Furthermore, ROI scalable video coding (SVC) also benefits from the proposed SVSP due to its hierarchical nature, as multiple perceptually prioritized ROIs can be extracted by simply segmenting the SVSP in multiple thresholds in a scalable manner. It can be applied to automatically evaluate the quality for ROI scalability, and thus has the potential to reduce the workload of subjective tests for ROI SVC algorithms in the current MPEG activities. Extensive experiments are conducted to show the performances that justify the effectiveness of the proposed SVSP model.
\end{abstract}

\section{INTRODUCTION}

Visual attention is one of the most important mechanisms of the human visual system (HVS), and computational visual attention models have been developed over the last 20 years and have already facilitated various aspects of the evolution in visual communication systems. One of its important applications is to enhance the image and video compression algorithms perceptually [1]. It is applicable to automatic spatiotemporal prioritization of arbitrary video contents for compression by validating their visual attention model into MPEG-1 and MPEG-4 [2].

Recently, scalable video coding (SVC) is being developed to encode video signal once, but enables decoding from partial streams with respect to the specific rate and resolution required by a certain application. Such scalability has important applications in many areas with heterogeneous networks, where downstream client capabilities and network conditions are not known in advance. Besides flexible spatial, temporal and signal-to-noise ratio (SNR) scalability, scalable coding shall support a mechanism that permits region-of-interest (ROI) scalability. For instance, a user may desire that a certain region in video sequence should be displayed at a higher quality than the rest of the video. ROI scalability enables the user

This work was supported by National Natural Science Foundation of China (60332030, 60502034, 60625103), Shanghai Rising-Star Program (05QMX1435), Hi-Tech Research and Development Program of China 863 (2006AA01Z124), NCET-06-0409, and the 111 Project. to preferably obtain the higher quality at the desired region under the limit of the available bandwidth. As an application of visual attention model, it is straightforward to incorporate a single topographic saliency map of visual attention that indicates the sensitivity of every location in an input image into non-scalable ROI coding [3]. However, ROI scalable coding requires the model to offer more flexibilities, since it may need saliency maps under various spatial/temporal resolutions instead of one to facilitate the coding process.

In [4], we propose a computational model for scalable visual sensitivity profile (SVSP), i.e., a hierarchy of saliency maps that simulate the bottom-up and top-down attention of the HVS. In this paper, we apply the proposed SVSP to image/video processing. It can detect the most significant visual information in a picture and spatially prioritize the information to augment the visual quality, such as in noiseshaping and JPEG2000 ROI coding. Furthermore, ROI scalable video coding also benefits from the proposed SVSP due to its hierarchical nature, as multiple perceptually prioritized ROIs can be extracted by simply segmenting the SVSP in multiple thresholds in a scalable manner.

The rest part of the paper is organized as follows. To better illustrate the application of the scalable visual sensitivity profile, a brief review of the model is given in Section II. The performance of the proposed SVSP in noise-shaping is presented in Section III, as an example to validate the model, followed by its application in JPEG2000 ROI coding in Section IV. Section V demonstrates its most characterized use in ROI scalable video coding in detail. And finally Section VI concludes the paper.

\section{Scalable Visual Sensitivity Profile}

We propose a computational model for scalable visual sensitivity profile in [4]. It is a two attentive-stage model based upon Treisman's pioneering work on visual attention [5], which divides the process into the pre-attentive stage and attentive stage. Meanwhile, it adds the scalable extension to generate a hierarchy of saliency maps, so that it can facilitate the ever-growing application in scalability.

The pre-attentive stage, which can also be referred as bottom-up attention stage, extracts low-level visual features such as intensity, color, orientation and movement and integrates them into a saliency map. This stage is stimulus driven. For this stage, we extend Itti's receptive field profile computation [3] into a full center-surrounded structure, so as 


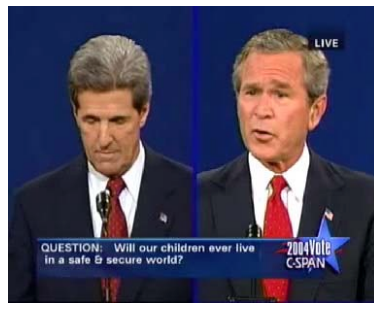

(a)

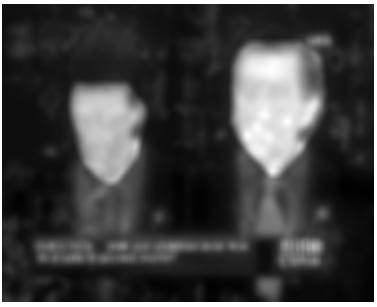

(b)
Fig. 1. (a) Frame 51 of video clip president debate. (b) SVSP with $l=0$.

to provide hierarchical saliency maps to be used in scalable coding. And we use Nothdurft's nonlinear addition model [6] to integrate low-level stimulus features to account for possible overlap between feature maps and generate more accurate saliency prediction. Let the pyramidal bottom-up attention profile be $B A P^{(l)}$, where $l \in[0, L]$ is the scalable level available.

The attentive stage, or the top-down stage, involves much more complex psychological process, and directs attention into certain objects within the scene. And this stage is knowledge driven. Compared with bottom-up attention, the investigation on top-down attention lays its emphasis on the top-down modulation processing on bottom-up features of visual attention [3]. It is widely known that human face and captions in scene often indicate useful recognition clues and attract knowledge-driven human attention. Consequently, in the current implementation, we use face and caption as topdown attention directors. And these top-down feature maps also take a hierarchical shape to be seamlessly combined with the afore-computed bottom-up features. Let the generated pyramidal profiles corresponding to skin map and caption map be $S P^{(l)}$ and $C P^{(l)}$ respectively.

With the hierarchical bottom-up and top-down saliency maps, we can integrate them into a SVSP

$$
V S P^{(l)}=B A P^{(l)} \cdot \alpha^{S P^{(l)}} \cdot \beta^{C P^{(l)}} l \in[0, L]
$$

where $\alpha, \beta \geq 1$ are weighting coefficients that indicate the influence of each top-down feature modulating to bottom-up attention map. Fig.1 presents an example of the generated SVSP with scalable level $l=0$ in 51th frame of president debate. The intensity indicates the visual significance of each location in the picture. The brighter part is more visually sensitive.

\section{SVSP IN NOISE SHAPING}

To validate the effectiveness of the proposed model, we first apply SVSP to noise shaping to show it improves visual quality by correctly extracting the perceptually significant information in a picture.

JND (Just-noticeable distortion/difference) refers to the visibility threshold below which changes cannot be perceived by human, due to the physiological and psychological imperfectness of the HVS [7]. It reveals the unevenness in processing ability of the HVS. Meanwhile, noise shaping is a popular way to evaluate the correctness of JND models. The reciprocal of JND profile can be regarded as the perceptual visual sensitivity, and vice versa. Therefore, the reciprocal of the proposed VSP (SVSP with scalable level $l=0$ ) can be considered as certain kind of "attention model-based JND" so as to be compared with other JND models. For an image frame $f_{i}(x, y)$ and its corresponding pixel-based JND model $J N D_{i}(x, y)$, the noise-injection process is

$$
\hat{f}_{i}(x, y)=f_{i}(x, y)+\tau S^{\text {rand }}(x, y) \cdot J N D_{i}(x, y)
$$

where $\tau$ is the constant used to adjust the amount of noise injected; theoretically if $\tau \leq 1$ the noise is perceptually unnoticeable, and $\tau>1$ the noise is noticeable. $S^{\text {rand }}$ takes +1 and -1 randomly. The proposed VSP-based JND model is defined as

$$
J N D^{V S P}(x, y)=\frac{\eta}{\xi+V S P(x, y)}
$$

where $\eta$ is a scaling coefficient to adjust the dynamic range so as to approach the actual JND threshold, and we simply set $\eta=1$ in this paper; $\xi$ is a small constant to avoid dividing by zero. We will compare it with Chou's JND model [8] $J N D^{C}$ and the JND model we previously proposed [9] $J N D^{Y}$. Since $J N D^{C}$ is merely defined for luminance of image, while $J N D^{Y}$ is defined in $\mathrm{YCbCr}$ space for image, for fairness, the experiments are conducted only on Y component, and the VSP in (2) uses no temporal information. The results are shown in Fig.2. As can be seen that the VSP-based JND outperforms $J N D^{C}$ and $J N D^{Y}$ in its ability to hide more noise in visually unimportant areas, while keeping the significant areas largely unaltered, which validates the proposed model to augment the visual quality. Note that although only VSP is presented in (3), the VSP-based noise-shaping algorithm can be easily extended to scalable noise-shaping due to its hierarchical nature.

\section{SVSP IN ROI CODING OF JPEG2000}

The proposed VSP can detect the most significant visual information in a picture and spatially prioritize the information to augment the visual quality. To this end, we validate our model in JPEG2000 coding standard, which supports both rectangular and arbitrary-shaped ROI coding without scalability, i.e. $l=0$ in SVSP. We define the arbitrary ROI in an image $R O I^{a}$ as areas that take half the top values in $V S P^{(0)}$

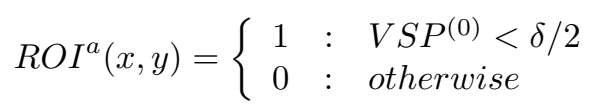

where $\delta / 2$ is half the dynamic range of $V S P^{(0)}$ and $x$ and $y$ are within the image dimensions.

To generate a rectangular ROI, we explore a seeded region growing algorithm [10]. The seed is placed at the most saliency point in $V S P^{(0)}$, and then expands to surroundings. The stopping criterion is that the pixel value on region borders falls below $60 \%$ of the starting seed-value. And the ROI is defined as the smallest rectangle that can hold the final result of the grown region. This ROI is denoted as $R O I^{r}$.

We test JPEG2000 ROI coding of both VSP-defined shapes in the frame image of President Debate, and ROI is extracted based on $V S P^{(0)}$ shown in Fig.1(b). As can be found in Fig.3, at the same bit-rate, the ROI aided coding takes superior qualities in both subjective and objective manner than coding without ROI. This indicates the information most significant to 


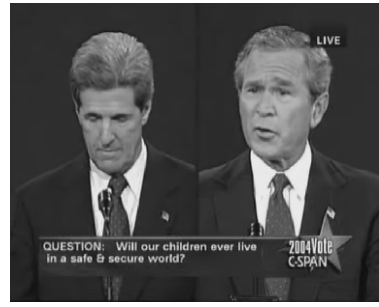

(a)

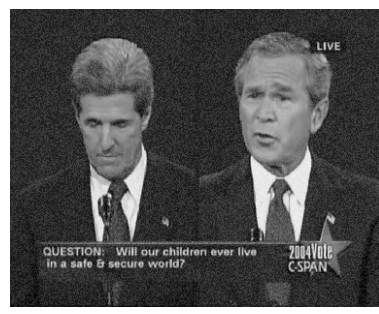

(c)

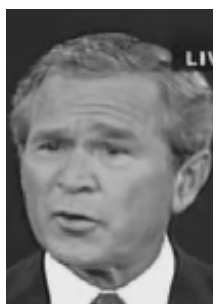

(e)

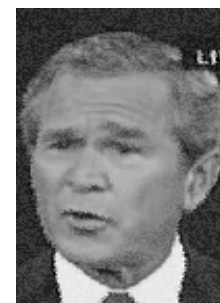

(f)

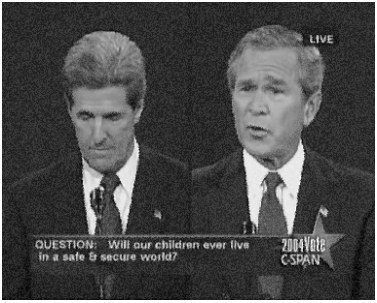

(b)

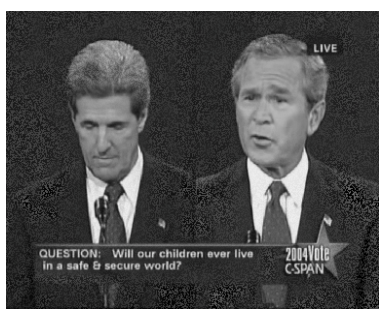

(d)

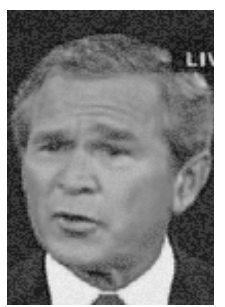

(g)

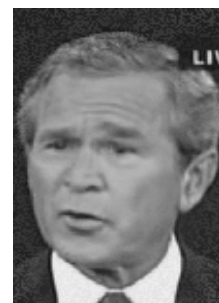

(h)
Fig. 2. (a) Luminance of frame 51 in president debate. (b) Noise-injected image with Chou's JND model, PNSR=25.99 dB. (c) Noise-injected image with Yang's JND model, PNSR=25.99 dB. (d) Noise-injected image with proposed VSP-based JND model, PNSR=25.99 dB. (e)-(h) are details of the most sensitive part (with the highest VSP values) of (a)-(d)

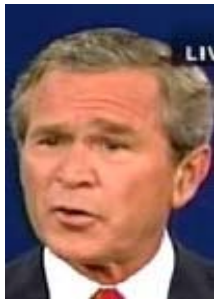

(a)

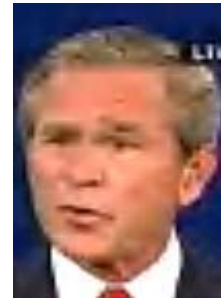

(b)

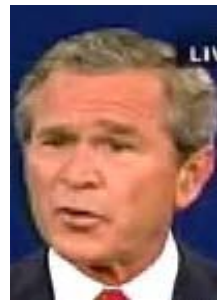

(c)

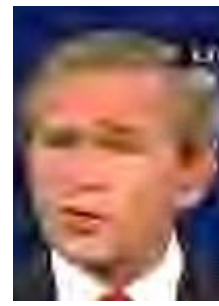

(d)
Fig. 3. (a) Details of the most sensitive part (with the highest VSP value) of frame 51 in president debate. (b) Details of image coded at $0.1 \mathrm{bpp}$ with arbitrary ROI defined in VSP, PSNR-Y=27.2dB. (c) Details of image coded at $0.1 \mathrm{bpp}$ with rectangular-shaped ROI defined in SVP, PSNR-Y=32.6dB. (d) Details of image coded at $0.1 \mathrm{bpp}$ without ROI, PSNR-Y=24.0dB.

human vision can be better preserved referring to VSP of the image at a certain bit restraint. We also note that rectangular ROI obtains better results than arbitrary-shaped ROI, in that it defines ROI in a more concentrated way so that bits can be assigned to one most significant part in an image rather than be distributed to several parts of less significance.

\section{SVSP in ROI SCALABLE VIDEO CODING}

The scalable video coding is currently being developed as an extension of the ITU-T Recommendation H.264/AVC [11]. Although the JSVM (Joint Scalable Video Model) reference software for the SVC project does not support ROI, several

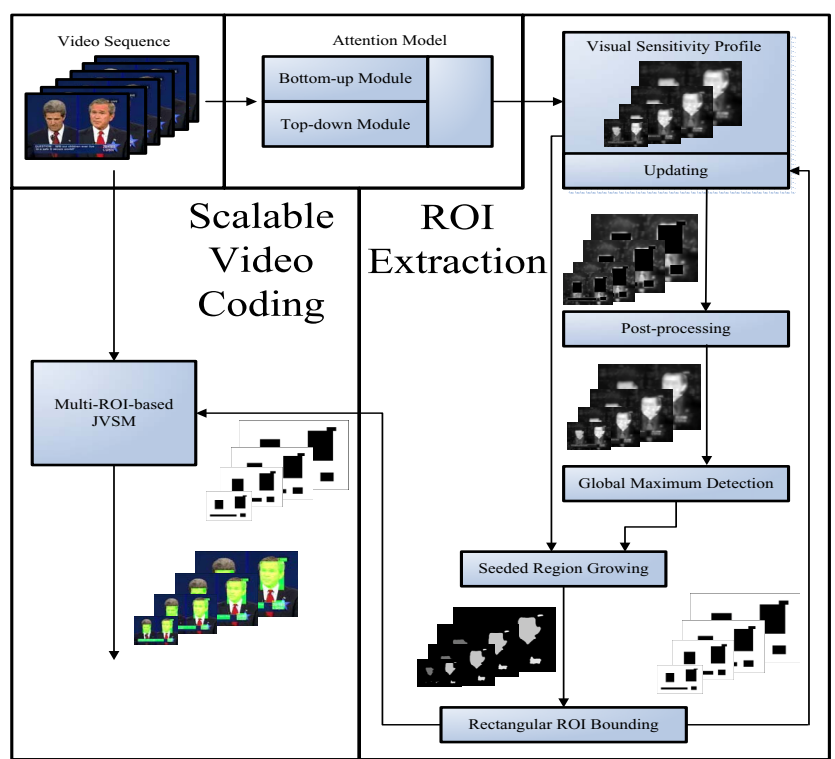

Fig. 4. Diagram of SVSP guided ROI scalable video coding.

proposals concerning the integration of ROI functionality into SVC have been put forward in the past JVT meetings [13] [?].

In this experiment, to demonstrate the application of the proposed SVSP to evaluate the quality for ROI SVC algorithms, we use JVT-R023 [12] as a reference software, and the diagram of ROI-based spatial scalable video coding is shown in Fig.4.

For one frame, it calculates its bottom-up and top-down attention profiles and integrates them into a SVSP with spatial scalable levels, and each level is then brought to an ROI extraction module. In this module, VSP of certain level is firstly smoothed in a post-processing stage to filter out isolated maxima, and then a global maximum detection is performed to find the most saliency point of the profile. The point is then used as a seed, and the seeded region growing algorithm is conducted within the VSP to find the most sensitive regions around the seed. The region expands until the average VSP value drops below $60 \%$ of the seed's value. And since the JVTR023 software only takes rectangular ROI, we use the smallest quadrangle containing the grown region as the ROI area. This ROI area is then cropped out from the original VSP in an update stage. Afterward, the seeded region growing algorithm is iteratively applied to the updated VSP until the multiple ROI areas of predefined number are all found.

The computed ROIs in a frame can be incorporated into the software coder as follows. In JVT-R023 configuration, there is an ROI description file for each spatial scalable layer, except for the base layer which is of the smallest spatial size. Since ROIs have been extracted ROIs in each level of SVSP that corresponds to a spatial scalable layer, we identify the required ROI parameters, i.e. ROI index to identify the ROI among multiple ROIs, up-left corner coordinate, width and height of the ROI area, and write the parameters into the layer's ROI description file. The coded bitstream can be truncated for scalable application. It is assumed that base layer can always be correctly decoded, so no ROI coding is supported in the 


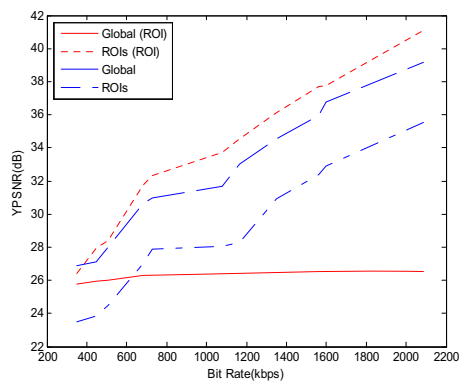

(a)

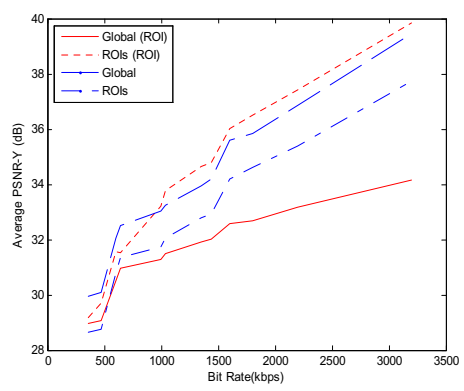

(c)

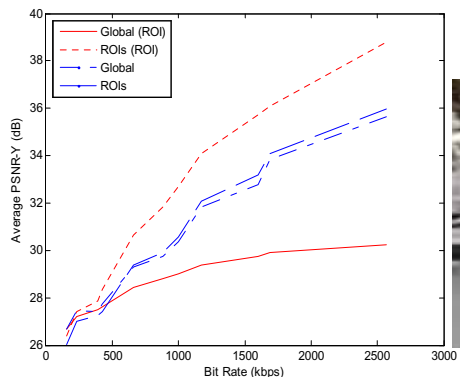

(b)

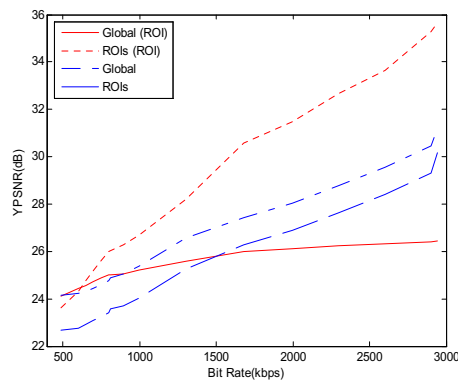

(d)

Fig. 5. (a) Average PSNR-Y vs. bit rate of president debate. (b) Average PSNR-Y vs. bit rate of foreman. (c) Average PSNR-Y vs. bit rate of crew. (d)Average PSNR-Y vs. bit rate of coastguard. In each figure, two red curves indicate global and ROI quality with SVSP, while two blue ones indicate global and ROI quality without ROI.

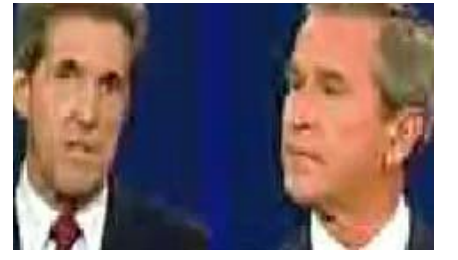

(a)

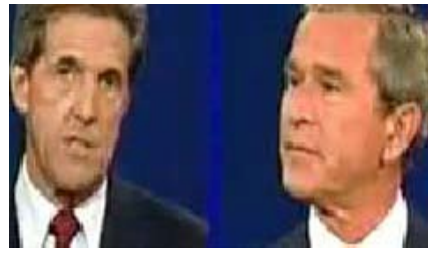

(b)

Fig. 6. Visual comparison in saliency area of frame 60 in president debate, CIF size coded at $900 \mathrm{kbps}$, (a)without ROI; (b)with SVSP defined ROI.

base layer. When the bit-rate truncation point exceeds that of the minimum base layer, the software makes sure that the nonROI data of the enhancement layer would be discarded first to prioritize the reconstruction of the most visual sensitive part of a frame.

Fig.5 demonstrates the performance of the proposed SVSP defined ROI SVC in standard test sequences president debate, foreman, crew and coastguard. The average PSNR of Y component vs. bit rate for global frame and ROI areas is illustrated in red curves, compared with the performance without ROI scalability in blue curves. Clearly, when the bit stream is truncated at certain rate, the decoded frame with the SVSP defined ROI coding effectively enhance the quality of ROI areas at the expense of some global PSNR compared to that without ROI coding. However, despite the global PSNR drops, the enhanced visual quality of the ROI regions bring more preferable perceptual results. Some detailed visual comparison results are shown in Fig.6 and Fig.7. The proposed SVSP can effectively identify the most saliency areas in each frame and mark them as ROIs, which will be assigned more bits and thus obtain superior visual effect as compared to SVC without ROI functionality.

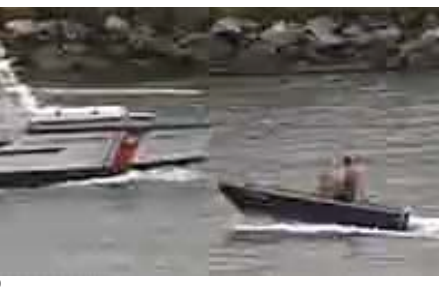

(a)

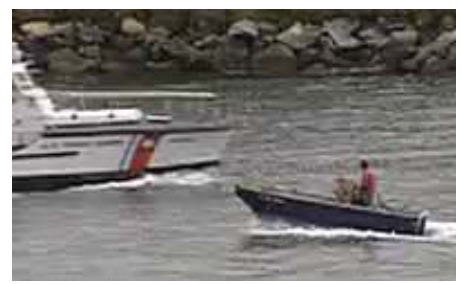

(b)
Fig. 7. Visual comparison in saliency area of the frame 27 in coastguard, CIF size coded at $850 \mathrm{kbps}$, (a)without ROI; (b)with SVSP defined ROI.

\section{CONCLUSION}

This paper applies the proposed computational model for scalable visual sensitivity profile (SVSP) to image/video processing. SVSP generates a hierarchy of saliency maps that simulate the bottom-up and top-down attention of the HVS. It can detect the most significant visual information in a picture and spatially prioritize the information to augment the visual quality, which has been verified in noise-shaping and JPEG2000 ROI coding. Furthermore, ROI scalable video coding also benefits from the proposed SVSP due to its hierarchical structure. Extensive experimental results have justified the effectiveness of the proposed SVSP model.

\section{REFERENCES}

[1] Z. Wang, L. Lu, and A. C. Bovik, "Foveation scalable video coding with automatic fixation selection," IEEE Transactions on Image Processing, vol. 12, no. 2, pp. 243-254, 2003.

[2] L. Itti, "Automatic foveation for video compression using a neurobiological model of visual attention," IEEE Transactions on Image Processing, vol. 13, no. 10, pp. 1304-1318, 2004.

[3] L. Itti, C. Koch, and E. Niebur, "Model of saliency-based visual attention for rapid scene analysis," IEEE Transactions on Pattern Analysis and Machine Intelligence, vol. 20, no. 11, pp. 1254-1259, 1998.

[4] G. T. Zhai, Q. Chen, X. K. Yang, W. J. Zhang,'Scalable Visual Significance Profile Estimation", submitted to International Conference on Acoustics, Speech, and Signal Processing, April, 2008, Las Vegas, US.

[5] A. M. Treisman and G. Gelade, "A feature-integration theory of attention," Cognitive Psychology, pp. 97-136, 1980.

[6] H.C.Nothdurft, "Saliency from feature contrast: additivity across dimensions," Vision Research, vol. 44, no. 10, pp. 1183-1201, 2000.

[7] W. S. Lin, "Computational Models for Just-Noticeable Difference," in Digital Video Image Quality and Perceptual Coding. H.R.Wu and K.R.Rao, Eds. Boca Raton,FL: CRC Press, 2006, pp. 281-303.

[8] C. H. Chou and Y. C. Li, "Perceptually tuned subband image coder based on the measure of just-noticeable-distortion profile," IEEE Transactions on Circuits and Systems for Video Technology, vol. 5, no. 6, pp. 467476, 1995.

[9] X. K. Yang, S. Yao, Z. Lu, W. S. Lin, and E. P. Ong, "Just-noticeabledistortion profile with nonlinear additivity model for perceptual masking in color images," in ICASSP, IEEE International Conference on Acoustics, Speech and Signal Processing - Proceedings, 3 ed Hong Kong, Hong Kong: Institute of Electrical and Electronics Engineers Inc., 2003, pp. 609-612.

[10] Anil K. Jain, Fundamentals of Digital Image Processing Prentice Hall, 1989.

[11] JVT-V021, Joint Video Team (JVT) of ISO/IEC MPEG \& ITU-T VCEG 22nd Meeting: Marrakech, Morocco, 2007.

[12] M. H. Lee, H. W. Sun, D. Ichimura, Y. Honda, and S. M. Shen, "CE4: Improved Interactive ROI Scalabilit," JVT-R023, Joint Video Team (JVT) of ISO/IEC MPEG \& ITU-T VCEG 18th Meeting: Bangkok, Thailand, 2006.

[13] T.C.Thang and et al, "Spatial Scalability of Multiple ROIs in Surveillance Video," JVT-O037, Joint Video Team (JVT) of ISO/IEC MPEG \& ITU-T VCEG 18th Meeting: Bangkok, Thailand, 2006. 